\title{
Analysis of Index Modulation of Doppler microembolic signals Part II: in vitro discrimination
}

\author{
Jean-Marc Girault, ${ }^{a}$ Denis Kouamé, ${ }^{\mathrm{b}}$ Sébastien Ménigot, ${ }^{\mathrm{a}}$ \\ Francesco Guidi, ${ }^{c}$ Grégory Souchon, ${ }^{\text {a }}$ Jean-Pierre Remenieras ${ }^{\text {a }}$ \\ "Université François Rabelais de Tours, UMRS "Imaging and Brain" U930 and CNRS ERL \\ 3106, Tours, France. \\ ${ }^{\mathrm{b}}$ Université PAUL Sabatier de Toulouse 3, IRIT UMR 5505, France \\ ${ }^{\mathrm{c}}$ University of Florence, Department of Electronics and Telecommunications, Italy
}

Corresponding Author: Jean-Marc Girault

Inserm U930 - CNRS ERL 3106 - Équipe 5

UFR de Médecine

10 boulevard Tonnellé, BP3223

F-37032 TOURS CEDEX 1

Email: jean-marc.girault@univ-tours.fr

Telephone: +33 (0)2 47366221

Elsevier

Ultrasound in Medicine \& Biology, Volume 37, Issue 1, January 2011, Pages 102-111

DOI : 10.1016/j.ultrasmedbio.2010.10.014

http://www.sciencedirect.com/science/article/pii/S0301562910005466 


\begin{abstract}
The purpose of this study was to validate through experiments that frequency modulation (FM) of microembolic signatures was principally due to the radiation force. Several experiments were required to prove that such a frequency modulation originates from micro-displacements induced by the radiation force acting on microbubbles. The first experiment was performed to verify that the diffraction effects due to the presence of a skull did not disturb the acoustic field appreciably and to validate that a radiation force in the brain was sufficient to create a detectable micro-displacement. A second in vitro experiment using a single gate transcranial Doppler (TCD) system was conducted to show discrimination feasibility and to check that microembolic frequency modulation signatures (FMS) and frequency modulation index (FMI) were the same as those observed in vivo and those calculated by simulation. A final in vitro experiment was performed using a multi-gate multi-channel TCD system to confirm the second experiment by directly measuring the micro-displacement induced by the radiation force. A new parameter, to be known as the position modulation index (PMI), is proposed. We showed that the radiation force is sufficient to induce detectable micro-displacements despite the presence of the skull. We also showed that the diffraction effects due to the skull induced a decrease in the ultrasound beam of 7.6 dB. Finally, we showed by using FMI and PMI that it is possible to discriminate gaseous from formed elements $(<100$ microns $)$ despite the presence of the skull. The discrimination based on the FMI is an off-line technique allowing the analysis of standard TCD recordings. However, discrimination based on the PMI requires recordings obtained exclusively from a multi-gate system.
\end{abstract}

Key words: Discrimination, Microemboli, ultrasound radiation force. 
More than two-thirds of all ischemic strokes are caused by cerebral embolisms related to the intravascular migration of various particles. These particles can be of different types, such as blood clots, fat particles or gas bubbles originating from the vascular bed or trapped in the vasculature during a surgical procedure or intervention. In order to provide an accurate diagnosis and suitable treatment, it is essential to establish the precise nature of emboli as quickly as possible. Embolus size is also an important criterion which must be taken into account. Transcranial Doppler (TCD) systems have been used in vivo, and various results have been reported in terms of detection rate and characterization. Analysis of the Doppler signal in well-controlled in vitro experiments can provide valuable information concerning the characteristics and size of emboli. However, accurate characterization cannot yet be fully achieved in clinical practice using current technology.

Many reports in the medical literature have proposed methods of differentiating gaseous and particle microemboli. Many in vitro and in vivo studies have been published in scientific and clinical journals (Rusell et al. (1992), Markus and Brown (1993), Georgiadis et al. (1994), Droste et al. (1994), Smith et al. (1997), Smith et al. (1998), Devuyst et al. (2000), Devuyst et al. (2001), to name but a few). They are mainly based on the measurement of duration (or conversely bandwidth) (Smith et al. (1997), Devuyst et al. (2001)), position-velocity-acceleration (or Doppler frequency, frequency modulation index, position modulation index) (Smith et al. (1997), Girault et al. (2010)) or a combination (SVL) (Smith et al. (1998)), and intensity or intensity ratio (Smith et al. (1997),Devuyst et al. $(2000))$.

The present study focused on the experimental measurement of the frequency modulation index (FMI) (Smith et al. (1998)), i.e. the slope of the embolus velocity (frequency modulation signature) versus time, and the position modulation index (PMI) (Girault et al. (2010)), i.e. the slope intensity of the position modulation signature. Our study 
complements and experimentally validates the simulation studies (Girault et al. (2010)) in which we argued that the major contribution of the frequency modulation signature (FMS) originates from an external force: the radiation force. However, this hypothesis can be questioned for many reasons, the main reasons being firstly the beam distortion due to the presence of the skull between the probe and the middle cerebral artery (MCA) and secondly the attenuation due to the depth of the region being explored $(>50 \mathrm{~mm})$. Note that the middle cerebral artery is the vessel most typically monitored for microemboli in current practice. Eighty percent of the flow to the brain goes through the left and right middle cerebral arteries, and thus most microemboli will be trapped here. When the middle cerebral artery is viewed from the temporal bone, the blood flow is mainly parallel to the ultrasound beam axis. A detailed discussion of the influence of the temporal bone on the US beam is presented in Deverson et al. (2000). However, we showed in this study that, although the US beam was attenuated and distorted, the radiation force induced was sufficient to move microbubbles.

The purpose of our study was to confirm experimentally that the radiation force is the main physical phenomenon which explains the frequency modulation observed in microembolic signals (MES) in vivo. As an illustration, Fig.1 shows temporal and time frequency representations of a gaseous microembolic Doppler signal from which FMI can be evaluated manually: $F M I=\Delta_{f} / \Delta_{t}=300 / 0.013=20 \mathrm{kHz} / \mathrm{s}$. According to Smith et al. (1997), the FMS reported in Fig.1 belongs to the third type of FM, i.e. with a rapid change. This confirms that the microembolus is of a gaseous nature. The question which remains is whether the radiation force is sufficient to create a micro-displacement detectable by a TCD system ? If the answer is positive, then differentiation between gaseous and formed elements is possible.

In this study, the issue was approached in two ways. First, the acoustic field was measured with and without a skull between the probe and area being explored. It was then shown that it is possible to discriminate a gaseous from a formed element (piece of pork) by using FMI and PMI. Finally, the most important parameters influencing the radiation 
force were examined.

56

57

58

\section{Materials and Methods}

Three series of measurements were performed.

The first and the second were carried out in our laboratory. The first experimental setup focused on mapping the ultrasound field refracted in water with and without a skull. The skull sample used was an adult skull fixed in formalin ex vivo (skull donated by the Anatomy Department of Bretonneau Hospital, Tours, France). The post mortem ex vivo human skull consisted of the right half of the skull. The gender and exact age of the cadaver were unknown. No visible damage or malformation was observed.

Using a commercial TCD system, the second study focused on measuring the frequency modulation index (FMI) by means of a time-frequency representation of the Doppler MES. Frequency and pressure levels (or voltage applied to the transducer) were constant.

The third series of measurements was carried out at the University of Florence with a customized multichannel multigate system (MCMG) allowing two representations (timefrequency and time-depth) of the microembolic Doppler signal.

\section{Mapping of the Pressure field}

The purpose of this experiment was to map the acoustic field in order to evaluate the influence of the skull on the refracted ultrasonic beam and to quantify the corresponding losses due to the presence of a human skull.

${ }_{4}$ The US field refraction induced by bone has been the subject of many studies (for some examples see: M.A. Moehring (1996), Deverson et al. (2000), Evans (2006)), and our work complementing these previous studies. This research has been crucial because it has been 
shown by Girault et al. (2010) that microembolus trajectories depend on both the shape of the US beam and the spatial velocity distribution.

The experimental setup consisted of a waveform generator (HP 3314 A) connected to an amplifier. The signal was sent to a transducer (Imasonic, $13 \mathrm{~mm}, 2 \mathrm{MHz}$ ) in contact with the skull. The ultrasonic wave transmitted through the skull was received by a hydrophone (Precision Acoustics Ltd) coupled to an amplifier (Gain $25 \mathrm{~dB}$ ). The transducer and the hydrophone were submerged in a water tank. The pressure levels were obtained at different positions using a displacement system (NTR system, resolution $0.5 \mathrm{~mm}$ ). The signal was digitized with an oscilloscope (Lecroy LT374M) and then sent to a computer through a GPIB link. A photograph of the experimental setup is presented in Fig.2. In the first experiments the pressure levels were obtained in water without the skull and with a degassed skull inserted between the transducer and the hydrophone.

To confirm the impact of the skull on the ultrasound beam (diffraction effect), we performed simulations based on a finite differences technique (details reported in references Calle et al. (2005); Souchon et al. (2006); Bastard et al. (2009)).

\section{In vitro measurement with TCD system}

The purpose of this experiment was to measure the presence of microembolic "additional acceleration" due to the radiation force acting on a microbubble through measurement of the frequency modulation index.

A diagram of the experimental setup is presented in Fig.3. A gear pump (4) circulates degassed water in a tygon tube (2) of $5 \mathrm{~mm}$ internal diameter, $5 \mathrm{~mm}$ being slightly larger than the mean diameter of $3.2 \mathrm{~mm}$ of the MCA, probably involving a lower beam refraction compared to that observed in vivo. This tube crosses a water tank (1) in which a transcranial Doppler transducer (3) is immersed. Circulating emboli are then detected by the pulsed wave (PW) Doppler system (5). In order to approximate the 
clinical context, we used two types of configuration: one without and one with a degassed skull inserted between the tube and the probe. The transcranial Doppler transducer used was an unfocused Vermon probe of $2 \mathrm{MHz}$ central frequency. The PW Doppler system (DOP248 Ultrasons Technologies, Tours, France) operates at $2 \mathrm{MHz}$ with pulses of $N=20$ cycles (for example), a pulse repetition frequency (PRF) of $8 \mathrm{kHz}$ and an acoustic intensity of $I_{\text {spta }}=450 \mathrm{~mW} / \mathrm{cm}^{2}$. The corresponding pressure level at this intensity is:

$$
P=\sqrt{2 Z \frac{I_{s p t a}}{N} \frac{f}{P R F}}=400 k P a,
$$

where $P R F=8 \mathrm{kHz}, f=2 \mathrm{MHz}$ and $N=20$, and where the acoustic impedance in water is $Z=1.48$ MRay and in the brain is $Z=1.6$ MRay. Note that $I_{\text {spta }}=720 \mathrm{~mW} / \mathrm{cm}^{2}$ is the upper-end value under the FDA guidelines.

Bubbles were produced naturally by cavitation in the gear pump used to circulate water and they were all less than $300 \mu m$ in size. Note that the size of the microbubbles was measured on the basis of photographs where both microbubbles and reference objects of known size were present (micropipette, US probe). The solid emboli were pieces of pork (bought from a butcher) and they were all less than $300 \mu m$. Note that the size of the fat particle was measured with a binocular magnifying glass. We believe that $300 \mu \mathrm{m}$ is a value close to clinical situations. The velocity of the fluid in the tube was set at a constant value to ensure no acceleration. Solid microemboli were injected into a second water tank (6) and sucked into the tube. Gaseous and solid microemboli were detected when they crossed the ultrasound beam, and each microembolus signal was computed.

In order to quantify the frequency modulation, we introduced the Frequency Modulation Index (FMI), initially proposed by Smith et al. (1997). The FMI is defined as follows:

$$
F M I=\frac{d F_{D}(t)}{d t}=\frac{2 f \cos \theta}{c}\left(\frac{d V_{b}(t)}{d t}+\frac{d V_{R F}(t)}{d t}\right)
$$

where $F_{D}(t)$ is the instantaneous Doppler frequency, $V_{b}(t)$ the embolus velocity and $V_{R F}(t)$ the additional velocity due to the radiation force ( $\theta$ is assumed to be constant). In practice, 
$\widehat{F M I}$ was obtained manually (by means of a marker cursor) by measuring:

$$
\widehat{F M I}=\frac{\Delta_{f}(t)}{\Delta_{t}}=\frac{F_{D}\left(t-\Delta_{t} / 2\right)-F_{D}\left(t+\Delta_{t} / 2\right)}{\Delta_{t}},
$$

where $\Delta_{t}$ is the temporal interval in which the modulation is significant. Note that this approach is equivalent to applying a linear regression to the instantaneous frequency: the greater the pressure on the bubble, the higher the FMI.

\section{In vitro measurement with $M C M G$ system}

This experiment had two aims, the first being to verify that it was still possible to detect micro-displacements induced by the radiation force using another TCD system. The second aim was to measure the micro-displacement directly by means of a new parameter, the position modulation index (PMI). It should be noted that the FMI does not directly provide the value of the micro-displacement but yields an indirect value through the Doppler frequency.

A diagram of the experimental setup is presented in Fig.4. A micropipette (2) connected to a gas flow (3) (Eppendorf air generator) was immersed in a water tank. Microbubbles were produced at the extremity of the micropipette and rose to the surface of the water within the tank. An ultrasound beam (1) crossed the trajectory of the microbubbles. Acoustic emission $(2 \mathrm{MHz})$ and reception were performed with the MCMG board (4) connected to a computer. Bursts of 25 cycles were emitted with a PRF of $1 \mathrm{kHz}$. As the speed of the microbubbles was very slow in this in vitro experiment, we chose to reduce the commonly used PRF of $8 \mathrm{kHz}$ to $1 \mathrm{kHz}$. The direct consequence of this reduction was a lower total displacement of the microembolus. The value of the radiation force was not affected since the radiation force is independent of the PRF.

The MCMG board proposed by Guidi et al. (2003) provided acquisition of signals at 128 depths, the total depth covered being $128 c / 2 f_{s}$ where $c$ was the acoustic wave velocity and 
$f_{s}$ the fast time sampling rate programable in the range of 2-10 MHz. The signal detected at each depth was analyzed with the Wigner-Ville transform of blocks of 256 points obtained at $1 \mathrm{kHz}$ PRF. The frequency resolution of our representation was therefore $f=1.953 \mathrm{~Hz}$. With these parameters and an insonification angle of 45 degrees, the minimum velocity which could be measured was $v=1 \mathrm{~mm} / \mathrm{s}$. The temporal resolution was $1 / P R F=1 \mathrm{~ms}(P R F=1 \mathrm{kHz})$.

The MCMG board allowed direct display of the trajectory of an embolus crossing the ultrasound beam. It could also be used to compute the time-frequency representation from which the FMI could be evaluated. The magnitude of the displacement induced by the radiation force could be evaluated directly by measuring of the Position Modulation Index (PMI). In the case of a gaseous embolus, the Position Modulation Index (PMI) defined by:

$$
P M I(t)=\frac{d D(t)}{d t}
$$

where $D(t)$ is the instantaneous embolus position, could be determined from the timedepth representation by applying a linear regression to the trajectory of the embolus. Finally, in practice the evaluated $\widehat{P M I}$ was obtained manually by means of a marker cursor by measuring:

$$
\widehat{P M I}(t)=\frac{\Delta_{D}(t)}{\Delta_{t}}=\frac{D\left(t-\Delta_{t} / 2\right)-D\left(t+\Delta_{t} / 2\right)}{\Delta_{t}}
$$

\section{Results}

In this section we present the results obtained from in vitro measurements (see Figs.810). Three kinds of experiment were performed. The first involved measurements of the pressure level in water with or without a human skull. The second involved measurements of the frequency modulation index (FMI) with a classical TCD system, and the third involved measurements of FMI and PMI with a MCMG system. 
Mapping of the US beam

Figs. 5 and 6 illustrate the refracted acoustic field in water with and without the skull. The experimental mapping of the pressure level was obtained by measuring the maximum pressure at each position. The maximum pressure level measured in water without the skull was about $110 \mathrm{kPa}$, while with the skull the level measured was about $46 \mathrm{kPa}$. The presence of the skull induced a decrease of $7.6 \mathrm{~dB}$ and a lateral deviation (perpendicular to beam axis) of the ultrasound beam since the maximum pressure level was not at $0 \mathrm{~mm}$ (obtained without skull) but at $1.5 \mathrm{~mm}$. Note that this loss of $7.6 \mathrm{~dB}$ included diffraction effects, reflections and attenuation in water. Note also, as reported by Fry and Barger (1978), that slight defocusing and small reductions in beam occurred.

For example, for a fixed depth of $5.0 \mathrm{~cm}$ the spatial distribution of the pressure level at a fixed depth looked like a gaussian function (see Fig.2 in Deverson et al. (2000)). The $5.0 \mathrm{~cm}$ axial distance was chosen on the basis that most TCD recordings for the MCA are acquired at approximately this depth.

In two previous studies (Souchon et al. (2005) and Biard (2005)) we measured the pressure levels with a classical TCD system and with a hydrophone near the temporal bone, with and without a skull. The presence of the skull immersed in water caused a decrease in pressure of about $10 \mathrm{~dB}$ at $5 \mathrm{~cm}$ away from the skull compared to the $7.6 \mathrm{~dB}$ reported above.

Even when the pressure level fell drastically $(7.6 \mathrm{~dB})$, it was still possible to detect bubble displacement visually, as shown in Fig.7. Fig.7 shows a bubble flow when the trajectory deviated in the presence or absence of a skull between the bubbles and the transducer. In both cases the bubble flow deviated sufficiently to be observed by the human eye. 
Analysis of Frequency and position modulation

Figs.1 and 8 show temporal and time-frequency representations of in vivo and in vitro experiments, respectively. In these figures FMI were similar and of about $20 \mathrm{kHz} / \mathrm{s}$.

Fig.1 shows a time frequency representation of a gaseous MES obtained from the middle cerebral artery (depth $5 \mathrm{~cm}$ ). The setting parameters of the TCD system were $P R F=8$ $\mathrm{kHz}, N=20$ (number of cycles), $f=2 \mathrm{MHz}$, and $I_{\text {spta }}=450 \mathrm{~mW} / \mathrm{cm}^{2}$. Fig.1 shows temporal and time-frequency representations of an MES. Four phases can be seen in Fig.1 (bottom): i) constant speed of the bubble for a time position around $0.02 \mathrm{~s}$, ii) increasing speed between $0.02 \mathrm{~s}$ and $0.04 \mathrm{~s}$, iii) decreasing speed between $0.04 \mathrm{~s}$ and $0.06 \mathrm{~s}$, and iv) constant speed beyond $0.06 \mathrm{~s}$. The time-frequency embolic signature resembles a reversed "V" shape. Note that valid explanations of the "V" or reversed "V" shape are reported in Girault et al. (2010). The highest FMI was obtained in the decreasing phase where $\widehat{F M I}=$ $20 \mathrm{kHz} / \mathrm{s}$. The reversed "V" shape, which seemed to be representative of a gaseous MES, was similar to that reported by Smith et al. (1997) (for similar acquisition parameters) for which the FMI evaluated in the decelerating phase was $\Delta_{f} / \Delta_{t}=800 / 0.020=40$ $\mathrm{kHz} / \mathrm{s}$. Finally, when the time-frequency signature was monotonic, the FMI was simply evaluated by measuring the slope. When the time-frequency was no longer monotonic, the signature was split into two monotonic regions for which only the maximum slope was retained. Note also that for greater time-frequency resolution we used the Wigner-Ville representation, as suggested in Smith et al. (1997). Note that the scalloping effect present in Fig.8b is due to the inner interference of the Wigner-Ville transform.

Fig. 8 shows a time frequency representation for two types of embolus (gaseous and fat particle). Two time-frequency representations and two temporal representations of Doppler MES obtained from the experimental setup depicted in Fig.3 are presented in Fig.8 as examples. The bottom image, obtained for a solid embolus, did not show any frequency modulation (type I FM) whereas the top image, obtained for a gaseous embolus, demon- 
strated detectable frequency modulation (type III FM). Note that the temporal Doppler signature presented in Fig.8, obtained for emboli circulating in water, was very similar to that reported by Smith et al. (1997) obtained for emboli circulating in blood.

Fig.9 shows the time-depth representation of four gaseous emboli (evaluated radius of $\approx 200 \mu \mathrm{m}$ ) circulating in water obtained with the MCMG system as described in the experimental setup in Fig.4. The setting parameters were $f=2 \mathrm{MHz}, P R F=1 \mathrm{kHz}, N=$ 25 , and $P a=500 \mathrm{kPa}$. The position modulation index was $\Delta_{D} / \Delta_{t}=(180-80) /(3) \approx 30$ $\mathrm{mm} / \mathrm{s}$. This implies that the mean elementary displacement between two consecutive emitting bursts is about $30 \mu \mathrm{m}(P M I=30 \mathrm{~mm} / \mathrm{s}=30 \mu \mathrm{m} / \mathrm{ms})$ for a $P R F=1 \mathrm{kHz}$. With the depth resolution of $(200-60) / 128 \approx 1.1 \mathrm{~mm}$, microdisplacements of 30 microns were detected each $30 \mathrm{~ms}$. In terms of the shape of the microbubble trajectory, it can be seen in Fig.9 that the microbubble deviates more and more as it crosses the US beam: the closer the bubble approaches to the focal point, the more the bubble accelerates, and the further the bubble moves away from the focal point the more it slows down. The inflection point of the microbubble trajectory corresponds to the point where the pressure is maximal. This is easily explained by supposing a unimodal US beam profile.

\section{Discrimination}

FMI assessed from the experimental setup of Fig.4 are shown in Fig.10 using a classical TCD system for all gaseous and solid emboli (with and without a skull). Each cross in Fig.10 represents one measurement. Several crosses are superimposed, and the mean value of these measurements is represented by a circle. Measurements were very close in each configuration, allowing easy discrimination of an embolus (bubble or fat).

- For a microbubble and a pressure level of $500 \mathrm{kPa}$ (measured without skull), it can be seen that the FMI measured was around $10 \mathrm{kHz} / \mathrm{s}(4<\widehat{F M I}(\mathrm{kHz} / \mathrm{s})<40)$, whereas for a pressure level of $150 \mathrm{kPa}$ (with skull), the FMI was around $0.8 \mathrm{kHz} / \mathrm{s}(0.2<$ 
$\widehat{F M I}(\mathrm{kHz} / \mathrm{s})<2)$.

- Similarly, for a fat particle and a pressure level of $500 \mathrm{kPa}$ (measured without skull), it can be seen that the FMI was around $0.2 \mathrm{kHz} / \mathrm{s}(60<\widehat{F M I}(\mathrm{~Hz} / \mathrm{s})<600)$, whereas for a pressure level of $150 \mathrm{kPa}$ (with skull), the FMI measured was around $10 \mathrm{~Hz} / \mathrm{s}$.

FMI and PMI assessed from the experimental setup in Fig.3 using a MCMG TCD system for all gaseous and solid emboli are shown in Table 1. By using thresholds $(12.5 \mathrm{~Hz} / \mathrm{s}$ for FMI and $7 \mathrm{~mm} / \mathrm{s}$ for PMI), it was possible to discriminate gaseous from fat particles on the basis of FMI and PMI.

\section{Discussion and Summary}

The discussion focused on three essential areas. The first related to examination of the impact of a skull on the modification of an US beam and discussion of the visible deviation of microbubbles in the presence of a skull. The second area was the validation through in vitro experiments that the radiation force was the main physical phenomenon that explained the different type of FMS, focusing on qualitative findings concerning the similarities between our FMS results and those reported in the literature. The third area was the discrimination between gaseous and fat particles.

\section{Mapping of the US beam}

As reported by Fry and Barger (1978), and depicted here in Figs.5 and 6, the presence of the temporal bone distorted and attenuated the US beam.

In terms of beam distortion, the presence of a skull involves refraction of the ultrasonic beam, explained by the concave shape of the skull which induces mechanical focusing in some way. The maximum pressure was no longer $x_{1}=0 \mathrm{~mm}$ but $x_{1}=-1.5 \mathrm{~mm}$, proving that the beam was deflected (lateral deviation perpendicular to beam axis). Note that 
the principal lobe was distorted in its lower part both in simulation and experimentally. We hypothesized that this was due to the presence of the extra thickness of the skull bone, this extra thickness inducing a higher propagation velocity of the acoustic waves. In clinical practice, it is not possible to know the exact beam modifications due to the temporal bone. However, to overcome this lack of information, experts naturally and spontaneously correct the beam deflection by modifying the beam-to-flow angle and the depth of the Doppler window. On the other hand, as it is not possible to correct the nonuniformity of the US beam, it is highly probable that the microbubble trajectory will remain nonuniform. However, the trajectory modification did not confirm such nonuniformity in our study (see Fig.7) since the microbubble trajectory seemed monotonic, thus suggesting that the US beam was not appreciably distorted.

In terms of attenuation, the presence of a skull induced reductions of $7.6 \mathrm{~dB}$ and $10 \mathrm{~dB}$ in another study performed by Souchon et al. (2005). This variability of pressure reduction was evidently due to the intra variability of skulls. However, it should not be concluded that the presence of a skull would involve a decrease in the order of $10 \mathrm{~dB}$ each time. Indeed, clinicians are well aware that the temporal window is very patient-dependent: someone with a thin skull will have less pressure reduction whereas others with a thick temporal bone will have considerable pressure reduction.

Another point requiring discussion was the spectacular deviation of the microbubble flow by the US beam. This result was very interesting because the deviation of the microbubble was still visible even when there was a human skull between the probe and the insonified area. This shift of about $4 \mathrm{~mm}$ (with skull) led us to believe that it would still be detectable by the TCD system in the presence of both skull and brain, bearing in mind that microdisplacements in the order of a few hundred microns can be detected by a Doppler system (see Girault et al. (2010)). 
The first finding to be discussed is the obvious resemblance between the FMS represented in Fig.1 and those reported by Smith et al. (1997), i.e. i) no modulation was present for type I FM, ii) gradual increase was observed over the whole duration of the microembolic Doppler signal for type II FM, and iii) rapid change was observed in a small percentage of the total durations of the Doppler microembolic signal for type III FM. Note that the acquisition parameters in our experiment were similar to those reported by Smith et al. (1997). The in vivo results obtained for gaseous microemboli were similar both qualitatively and quantitatively (i.e. in shape and in terms of FMI values) to those of "type III". The same applied for type I FM, i.e. for formed elements. This confirmed that type III FM found in the literature were also found in our in vivo study. Though the acquisition parameters were different in our in vivo and in vitro studies, the FMS were similar qualitatively, suggesting that the underlying physical phenomenon was probably the same.

We believe that it is possible to obtain type II FM for other experimental settings and for other microemboli (other materials)and that it is still possible to observe type II FM for larger microemboli. The microembolus must not be too large because there is a risk that it will be interpreted as a hazard to the patient.

\section{Discrimination}

The comments below on discrimination first address differentiation of microbubbles and microparticles of fat. We then discuss the results demonstrated in Figure 10 and in Table 1.

This experimental study showed that the temporal bone influences the US beam in a non-negligible manner. However, we also showed that the microdisplacements (few tens 
of microns) induced by the radiation force were sufficient to be detected by a TCD system.

Moreover this study confirmed experimentally that type I FMS are due to solid particles whereas type III FMS are exclusively due to gaseous microemboli. Finally, these in vitro studies confirmed that both FMI and PMI are suitable tools to differentiate gaseous from solid emboli, and we suggest that the discrimination techniques based on FMI and PMI measurement remain off-line techniques.

However, this research is the first step towards a clinical application, and complementary studies must be performed to explore and understand what really happens for large microemboli.

\section{Acknowledgements}

This study was supported by the European Union (UMEDS Project) and by the French Government (CASC Project). I wish to thank P. Tortoli for valuable discussions.

\section{References}

Bastard, C., Remenieras, J., Calle, S., Sandrin, L., 2009. Simulation of shear wave propagation in a soft medium using a pseudospectral time domain method. Journal of the Acoustical Society of America, 2108-2116.

Biard, M., 2005. Instrumentation and doppler signal analyze : study and characterization of emboli. Ph.D thesis, Tours, France.

Calle, S., Remenieras, J., Matar, O. B., Elkateb, M., F.Patat, 2005. Temporal analysis of tissue displacement induced by a transient ultrasound radiation force. Journal of the Acoustical Society of America, 2829-2840.

Deverson, S., Evans, D., Bouch, D., 2000. The effect of temporal bone on transcranial doppler ultrasound beam shape. Ultrasound in medecine and biology 26, 239-244.

Devuyst, G., Darbellay, G., Vesin, J.-M., Kemeny, V., Ritter, M., Droste, D., Moline, 
C., Serena, J., Sztajzel, R., Ruchat, P., ang G. Dietler, C. L., Ringelstein, E., 2001. Automatic classification of hits into artifacts or solid or gaseous emboli by a wavelet representation combined with dual gated tcd. Ultrasound in medecine and biology 27, 2803-2809.

Devuyst, G., Vesin, J.-M., Despland, P.-A., Bogousslavsky, J., 2000. The matching pusruit method: a new method of characterizing micro-emboli signals ? Ultrasound in medecine and biology 26, 1051-1056.

Droste, D., Markus, H., Nassiri, D., Brown, M., 1994. The effect of velocity on the appearence of embolic signals studied in transcranial doppler models. Stroke 25, 986-991.

Evans, D., 2006. Embolus differentiation using multifrequency transcranial doppler. Stroke 37, 1641.

Fry, F., Barger, J., 1978. Acoustic properties of the human skull. J. Acoust. soc. Am. 63, $1576-1590$.

Georgiadis, D., Mackay, T., Kelman, A., Grosset, D., Wheatley, D., Lees, D., 1994. Differentiation between gaseous and formed embolic materials in vivo. application in prosthetic heart valve patients. Stroke 25, 1559-1563.

Girault, J.-M., Kouame, D., Menigot, S., Souchon, G., Tranquart, F., 2010. Embolus discrimination by means of ultrasound radiation force part i: Analysis of the frequency modulation by means of simulations. Ultrasound in medecine and biology XX, XX-XX.

Guidi, F., Boni, E., Tortoli, P., 2003. Acoustic method for real-time visualization of microbubble movements and rupture. IEEE Ultrasonics symposium, 1183-1186.

M.A. Moehring, J.A. Ritcey, A. I., 1996. Sizing emboli using pulse doppler ultrasound-ii. effects of beam refraction. IEEE Trans on Biomed Eng, 581-588.

Markus, H., Brown, M., 1993. Differentiation between different pathological cerebral embolic materials using transcranial doppler in a in vitro model. Stroke 24, 1-5.

Rusell, D., Brucher, R., , Madden, K., Clark, W., Sanset, P., Zivin, J., 1992. The intensity of the doppler signal caused by arterial emboli depends on embolus type and size. Stroke $23,158-162$.

Smith, J., Evans, D., Bell, P., Naylor, R., 1998. A comparison of four methods for distin- 
guishing doppler signals from gaseous and particulate emboli. Ultrasound in medecine and biology 29, 1133-1138.

Smith, J., Evans, D., Naylor, R., 1997. Analysis of the frequency modulation present in doppler ultrasound signal may allow differentiation between partiulate and gaseous cerebral emboli. Ultrasound in medecine and biology 23, 727-734.

Souchon, G., Callé, S., Gomez, M., Remenieras, J., Girault, J., 2006. Etude numérique et expérimentale de la propagation d'onde au travers du crâne : Application à la détection d'emboles. Congrès Français d'Acoustique, Tours, France, 371-374.

Souchon, G., Girault, J.-M., Biard, M., Kouamé, D., Tranquart, F., 2005. Gaseous and solid emboli differentiation using radiation force. IEEE Ultrasonics symposium, Rotterdam, 2070-2073. 
1 Embolus differentiation based on FMI and PMI (MCMG system and experimental setup illustrated in Fig.3) for bubbles $R_{0} \approx 100 \mu \mathrm{m}$ and fat particles $100<R_{0}(\mu \mathrm{m})<300$ immersed in water. $I_{\text {spta }}=450 \mathrm{~mW} / \mathrm{cm}^{2}$, $P R F=1 \mathrm{kHz}, N=25, f=2 \mathrm{MHz}$. 


\section{List of Figures}

1 In vivo experiment (embedding medium: blood). Gaseous microembolic signature obtained from the middle cerebral artery (depth $50 \mathrm{~mm}$ ). Setting parameters: $P R F=2.7 \mathrm{kHz}, N=20$ (number of cycles), $f_{0}=2 M H z, I_{\text {spta }}=450 \mathrm{~mW} / \mathrm{cm}^{2}$. a) Temporal representation of a microembolic signal. b) Time frequency representation of the Doppler signal from which the FMI evaluated in the decelerating phase is $\Delta_{f} / \Delta_{t}=330 / 0.013=25 \mathrm{kHz} / \mathrm{s}$. The reversed "V" shape, which seems to be representative of a gaseous embolic signature, is similar to that reported by Smith et al. (1997) for which the FMI evaluated in the decelerating phase was $\Delta_{f} / \Delta_{t}=800 / 0.020=40 \mathrm{kHz} / \mathrm{s}$. and MCMG systems). 
$5 \quad$ Map of US field without skull. Simulated US beam (Top) and Measured US beam (bottom). The maximum pressure levels were reported for each location. The focal area was near the point $(0 \mathrm{~cm}, 4 \mathrm{~cm})$. The principal lobe and the secondary lobes can be recognized.

6 Mapping of US field with skull. Simulated US beam (Top) and Measured US beam (bottom). The maximum pressure levels are reported for each location. The focal area was near the point $(2.1 \mathrm{~cm}, 2.7 \mathrm{~cm})$. The principal lobe and the secondary lobes can be recognized.

7 In vitro experiment (embedding medium: water). Trajectory of bubble flow deviated by the US beam $(2 \mathrm{MHz})$. a) Without a skull the bubble displacement was a few centimeters and b) with a human skull the bubble displacement between the transducer and the bubble flow was a few millimeters. $P R F=8 \mathrm{kHz}, N=20, I_{\text {spta }}=450 \mathrm{~mW} / \mathrm{cm}^{2}$.

8 In vitro experiment in water without a skull. a) time representation of an MES, b) Time frequency representation (Wigner-Ville Transform for improved time-frequency resolution) of a Doppler signal. MES were obtained by means of a TCD system (Atys Medical) $(P R F=8 \mathrm{kHz}$, $N=20$ cycles, $\left.f=2 \mathrm{MHz}, I_{\text {spta }}=450 \mathrm{~mW} / \mathrm{cm}^{2}\right)$. Gaseous embolus $\left(R_{0} \approx 100 \mu \mathrm{m}\right)$ circulating in a tube filled with water (in vitro experiment depicted in Fig.3) (upper graph), $\Delta_{f} / \Delta_{t}=(600-200) / 0.02=20 \mathrm{kHz} / \mathrm{s}$. c) Time representation of an MES and d) Time frequency representation of an MES. A fat particle $\left(R_{0} \approx 300 \mu \mathrm{m}\right)$ (piece of pork) circulating in a tube filled with water (in vitro experiment depicted in Fig.3) (bottom graph), $\Delta_{f} / \Delta_{t} \approx 0 \mathrm{~Hz} / \mathrm{s}$. The speed of the fluid was identical for all experiments. 
Table 1

Embolus differentiation based on FMI and PMI (MCMG system and experimental setup illustrated in Fig.3) for bubbles $R_{0} \approx 100 \mu \mathrm{m}$ and fat particles $100<R_{0}(\mu \mathrm{m})<300$ immersed in water. $I_{\text {spta }}=450 \mathrm{~mW} / \mathrm{cm}^{2}, P R F=1 \mathrm{kHz}, N=25, f=2 \mathrm{MHz}$.

\begin{tabular}{|c|c|c|}
\hline type/parameter & FMI (Hz/s) & PMI $(\mathrm{mm} / \mathrm{s})$ \\
\hline \hline fat & 20 & 6 \\
\hline gas & 5 & 8 \\
\hline
\end{tabular}

$9 \quad$ Time-depth representation of four gaseous emboli (evaluated radius of $\approx 200 \mu \mathrm{m})$ circulating in water obtained with the MCMG system as described in the experimental setup in Fig. 4). $f=2 \mathrm{MHz}, P R F=1 \mathrm{kHz}$, $N=25, P a=500 \mathrm{kPa} . \Delta_{D} / \Delta_{t}=(180-80) /(3) \approx 30 \mathrm{~mm} / \mathrm{s}$. Note that the thickness of the vertical signature corresponds to $c t / 2=c N / f / 2=$ $1540 \mathrm{~m} / \mathrm{sx} 25 / 2 \mathrm{MHz} / 2 \approx 10 \mathrm{~mm}$.

10 Frequency modulation index (FMI) for gaseous and solid emboli in the presence or absence of a skull in water $(\eta=1 \mathrm{mPa})$. These measurements were obtained from the experimental setup illustrated in Fig. $4 .+=$ one measurement (several crosses can be superimposed), $o=$ mean of all measurements. $I_{\text {spta }}=450 \mathrm{~mW} / \mathrm{cm}^{2}, N=20, P R F=8 \mathrm{kHz}$. 


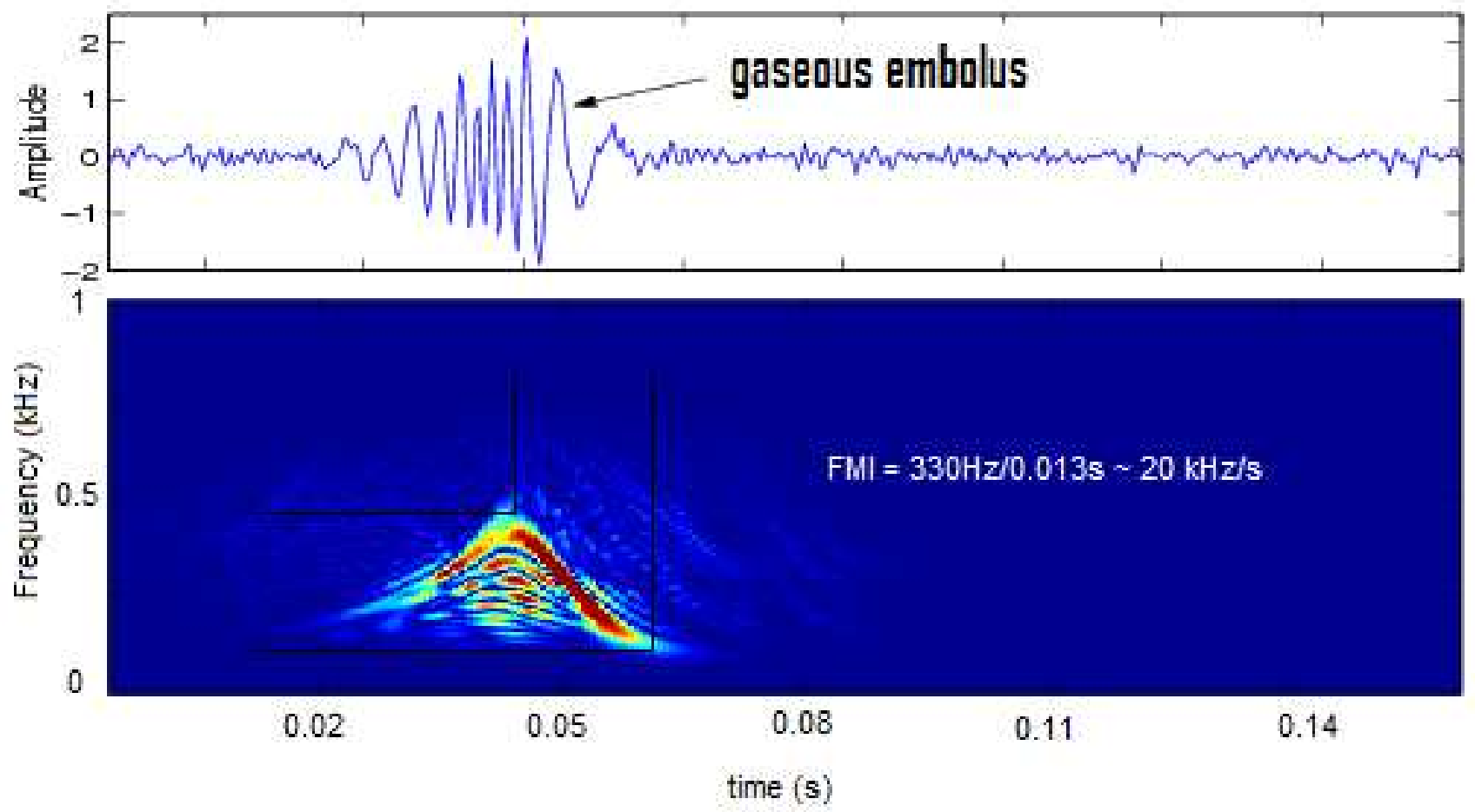

Fig. 1. In vivo experiment (embedding medium: blood). Gaseous microembolic signature obtained from the middle cerebral artery (depth 50mm). Setting parameters: $P R F=2.7 \mathrm{kHz}$, $N=20$ (number of cycles), $f_{0}=2 M H z, I_{\text {spta }}=450 \mathrm{~mW} / \mathrm{cm}^{2}$. a) Temporal representation of a microembolic signal. b) Time frequency representation of the Doppler signal from which the FMI evaluated in the decelerating phase is $\Delta_{f} / \Delta_{t}=330 / 0.013=25 \mathrm{kHz} / \mathrm{s}$. The reversed "V" shape, which seems to be representative of a gaseous embolic signature, is similar to that reported by Smith et al. (1997) for which the FMI evaluated in the decelerating phase was $\Delta_{f} / \Delta_{t}=800 / 0.020=40 \mathrm{kHz} / \mathrm{s}$. 


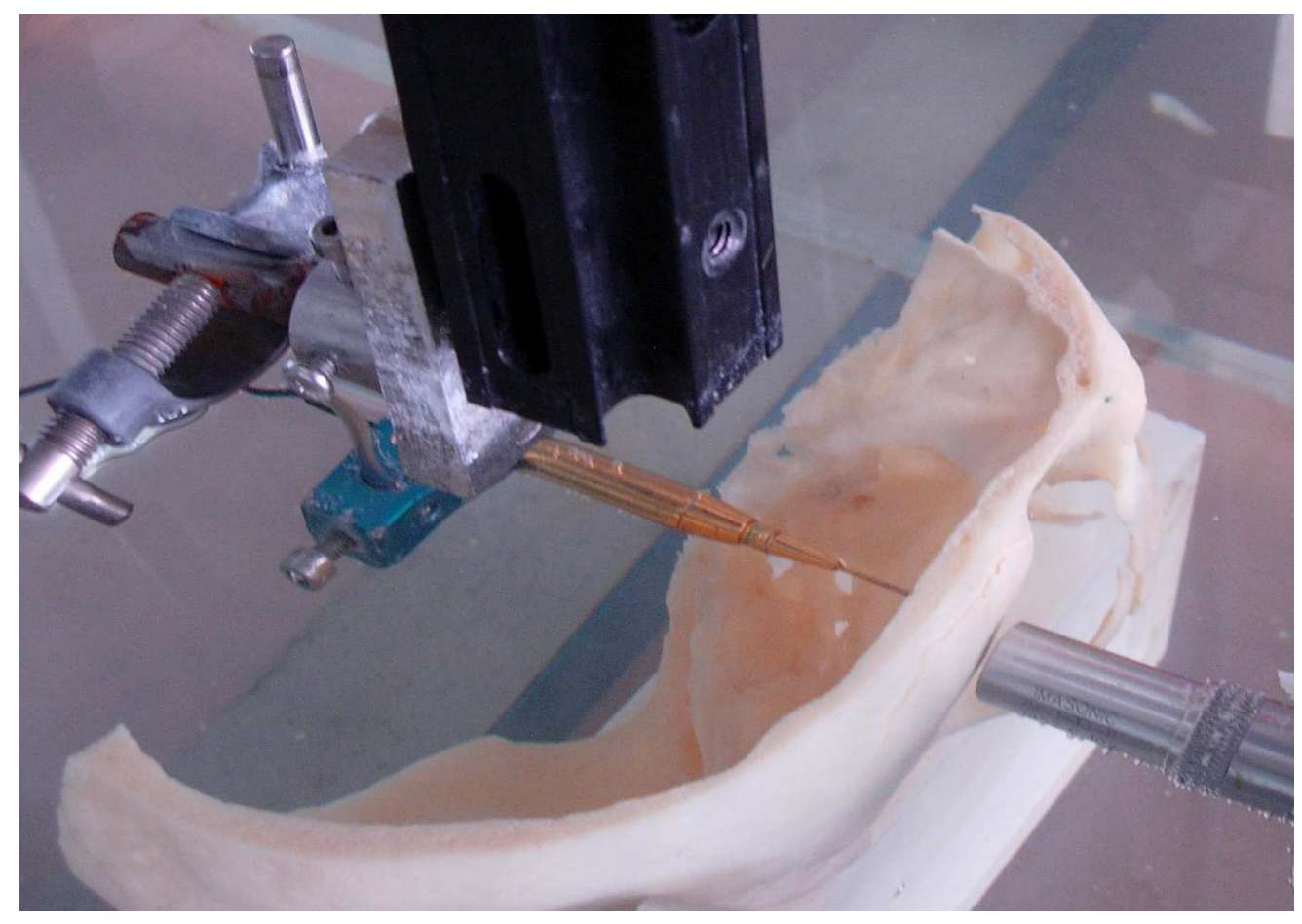

Fig. 2. Photograph of the experimental setup required to map the acoustic field.

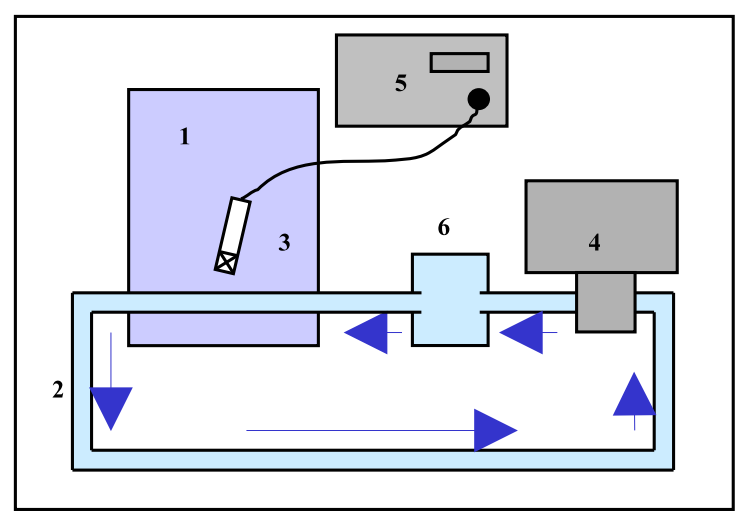

Fig. 3. Diagram of experimental setup. A gear pump (4) circulates degassed water in a tygon tube (2). This tube crosses a water tank (1) in which a transcranial Doppler transducer (3) is immersed. Circulating emboli are thus detected by the pulsed wave (PW) Doppler system (5)(usual TCD and MCMG systems). 


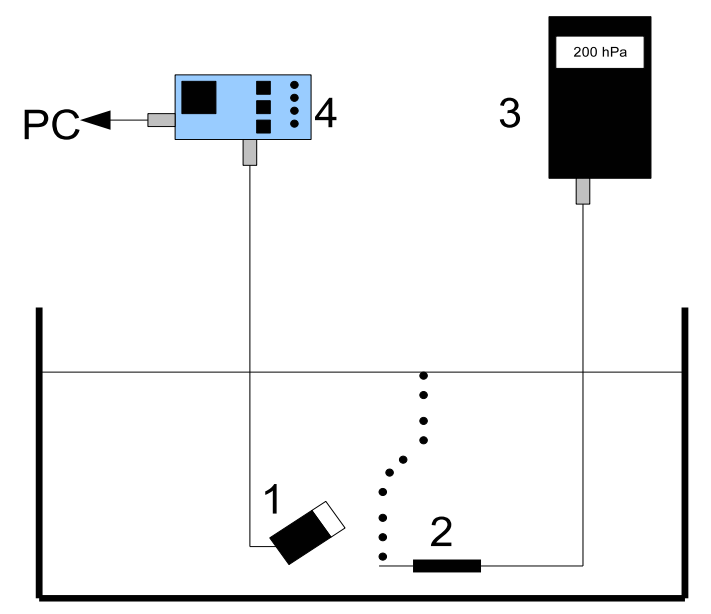

Fig. 4. Diagram of experimental setup. A micropipette (2) connected to a gas flow (3) (Eppendorf air generator) was immersed in a water tank. Bubbles were produced at the extremity of the micropipette and rose to the water surface within the tank. An ultrasound beam (1) crossed the trajectory of the bubbles. Acoustic emission and reception were performed by the MCMG system (4) connected to a computer. 


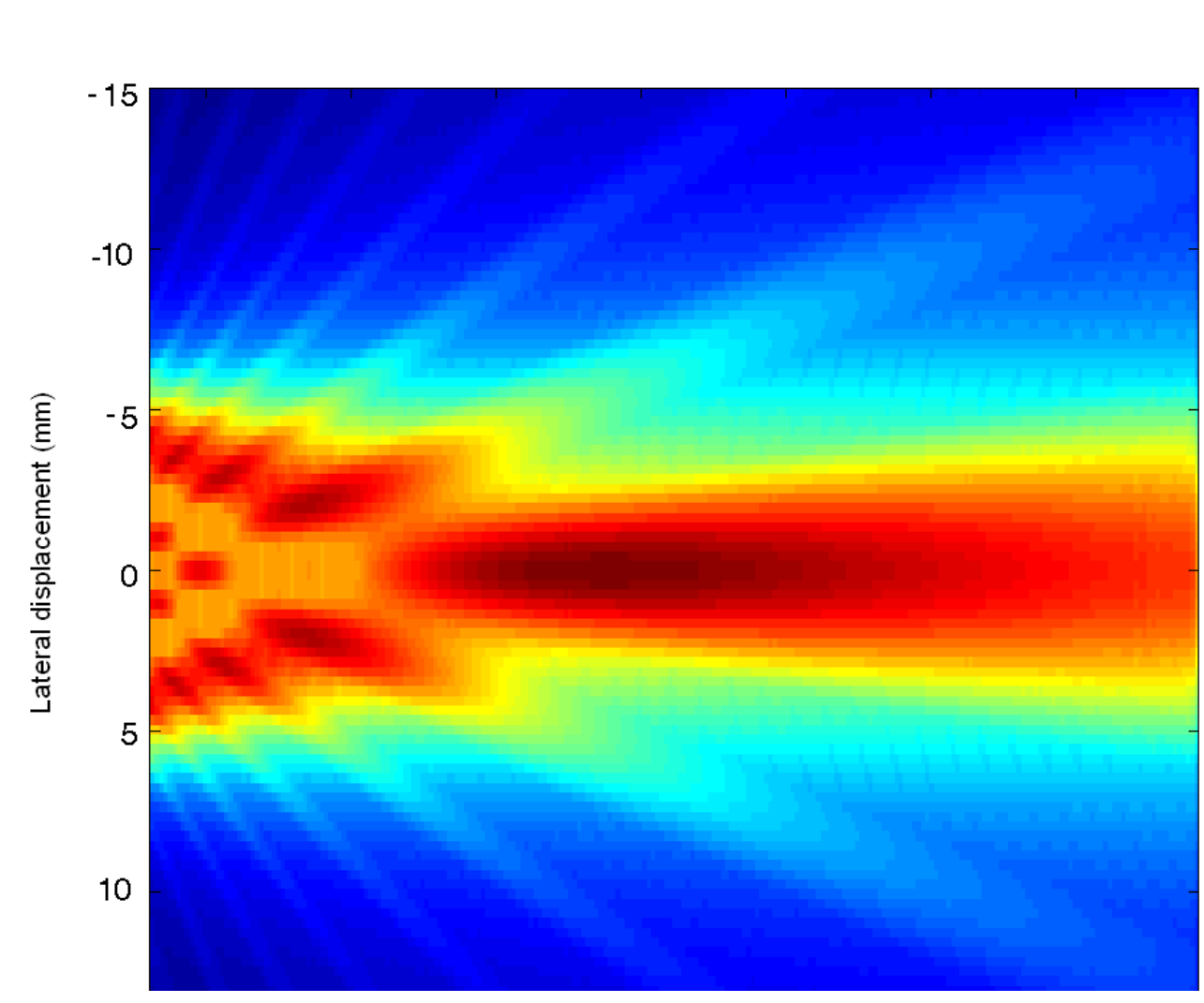

$\mathrm{Pa}$
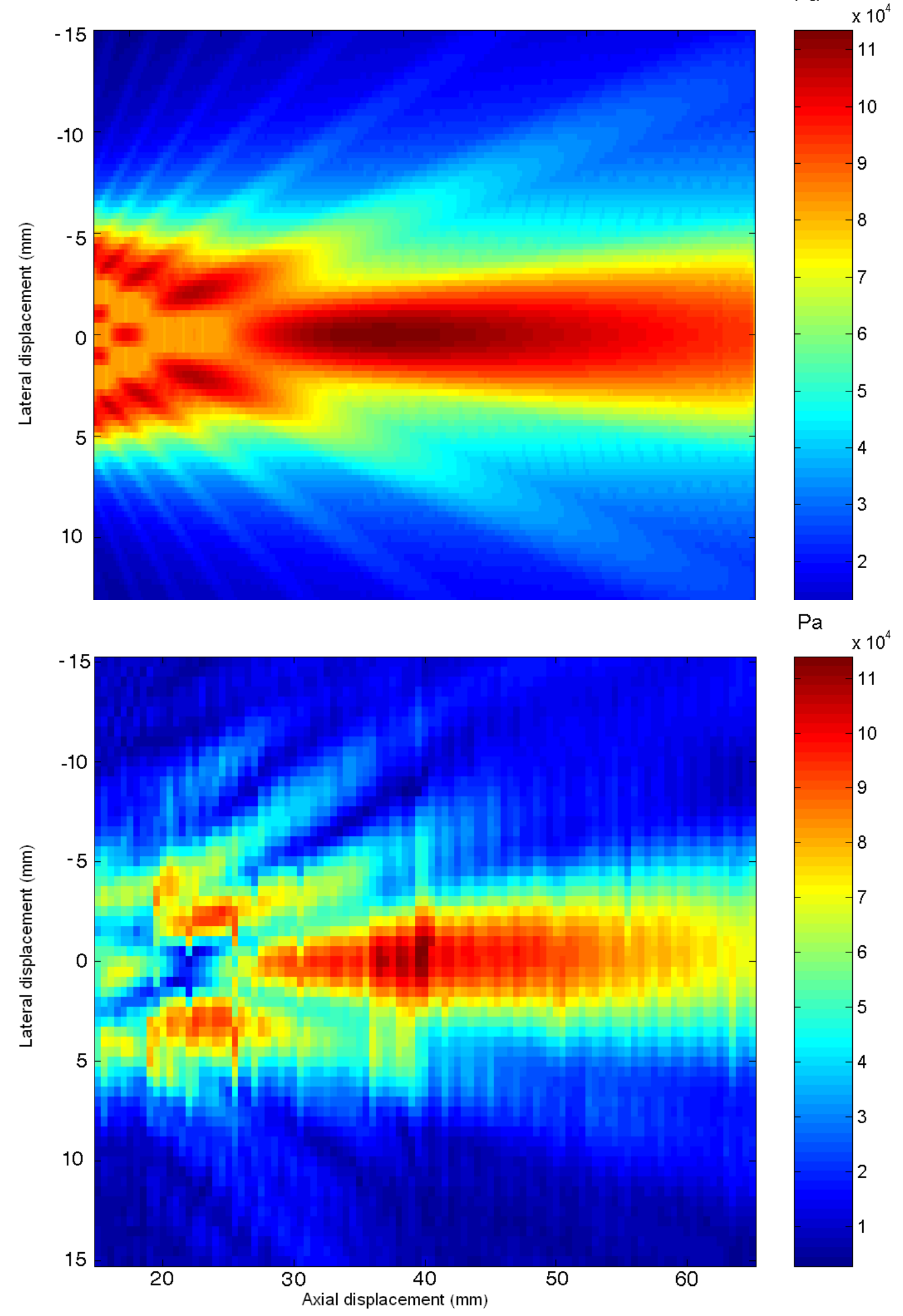

Fig. 5. Map of US field without skull. Simulated US beam (Top) and Measured US beam (bottom). The maximum pressure levels were reported for each location. The focal area was near the point $(0 \mathrm{~cm}, 4 \mathrm{~cm})$. The principal lobe and the secondary lobes can be recognized. 


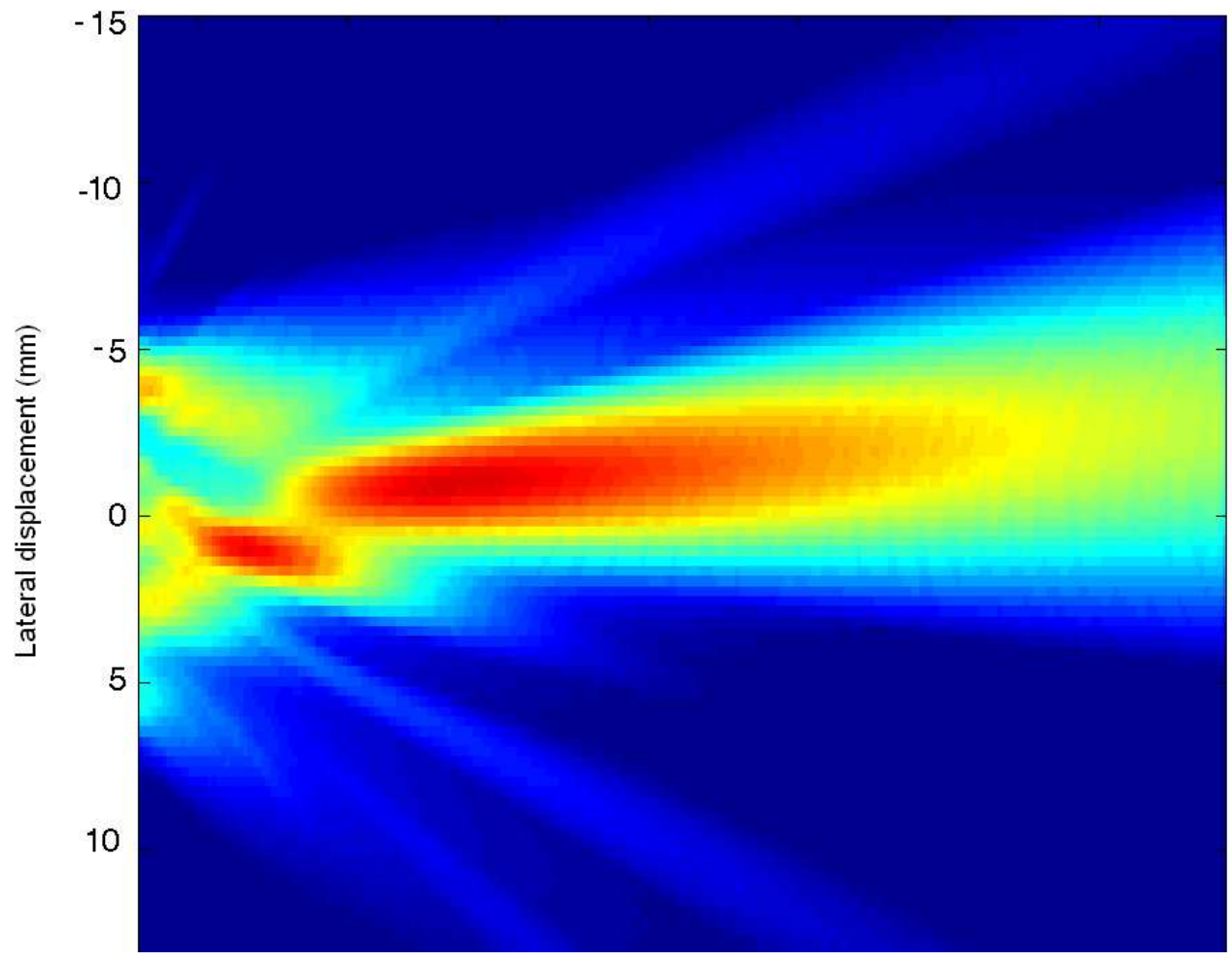

$\mathrm{Pa}$

$\times 10^{4}$
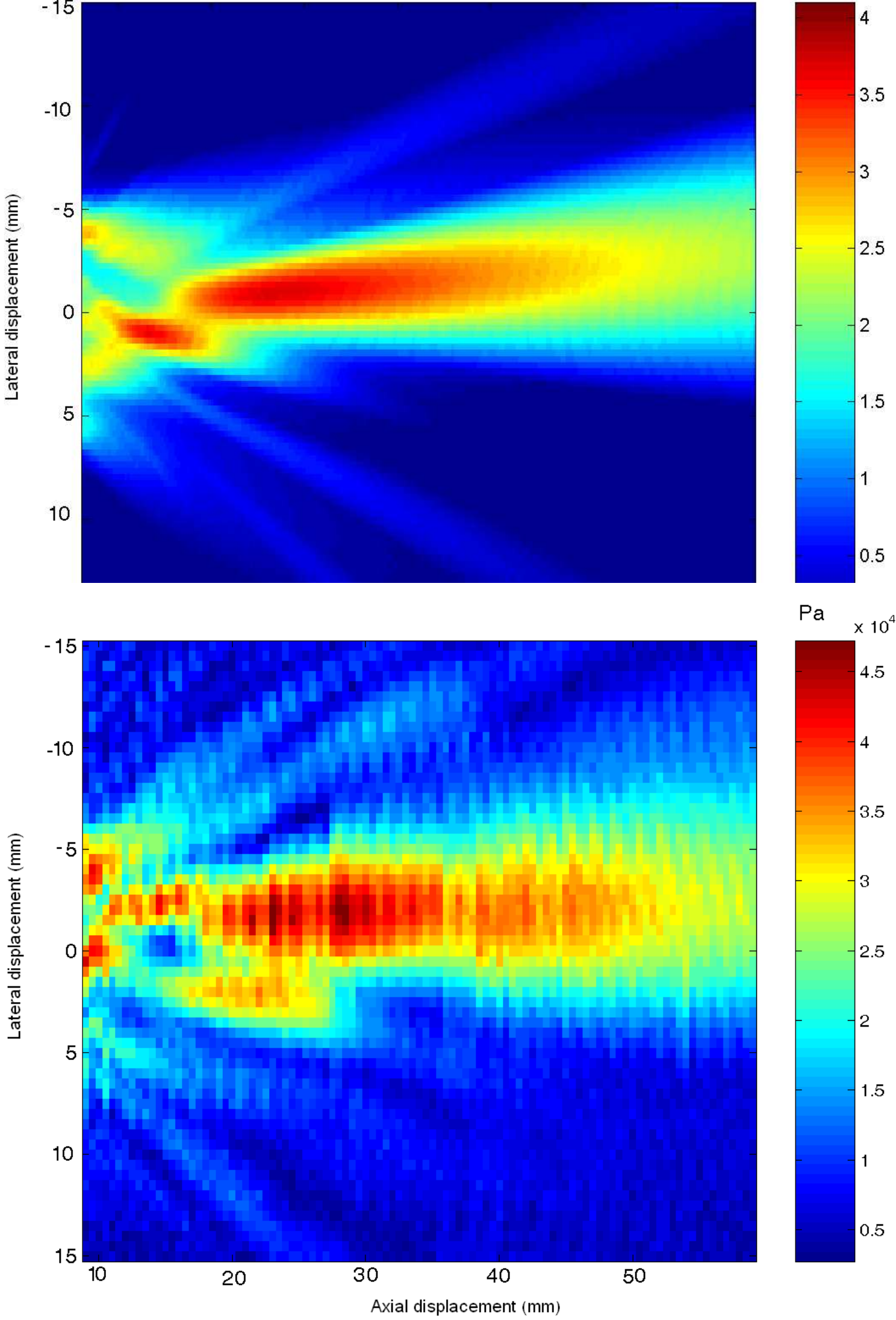

$\mathrm{Pa}$

Fig. 6. Mapping of US field with skull. Simulated US beam (Top) and Measured US beam (bottom). The maximum pressure levels are reported for each location. The focal area was near the point $(2.1 \mathrm{~cm}, 2.7 \mathrm{~cm})$. The principal lobe 27 and the secondary lobes can be recognized. 

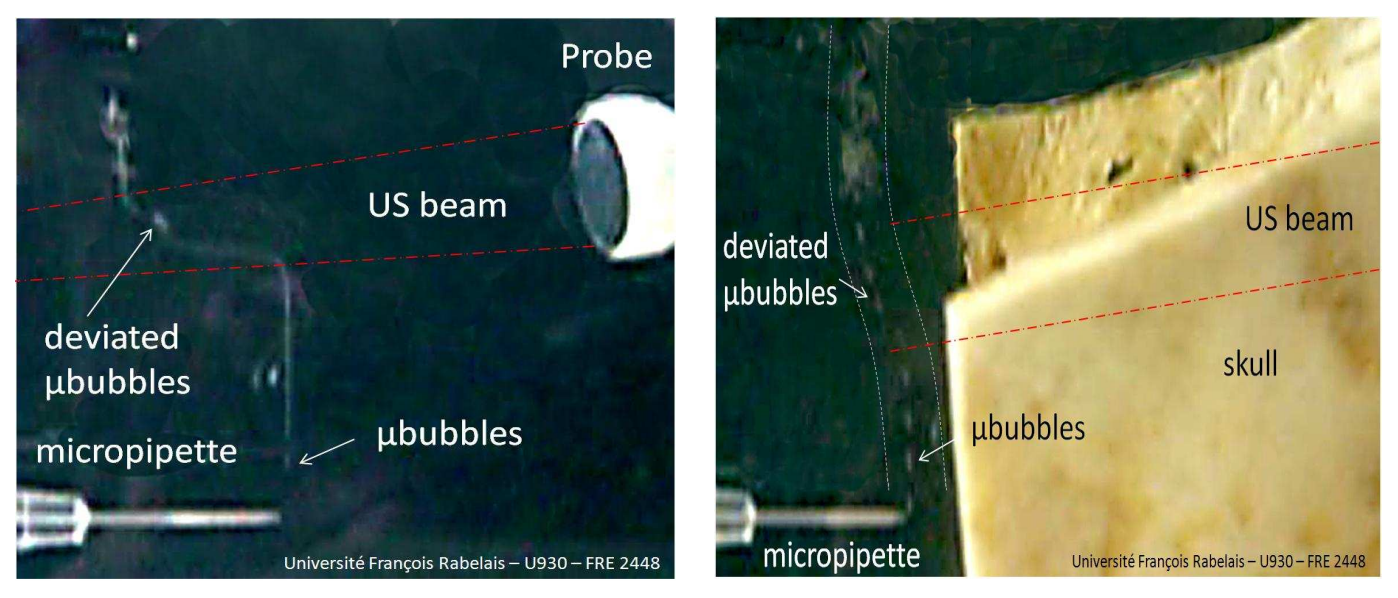

Fig. 7. In vitro experiment (embedding medium: water). Trajectory of bubble flow deviated by the US beam $(2 \mathrm{MHz})$. a) Without a skull the bubble displacement was a few centimeters and b) with a human skull the bubble displacement between the transducer and the bubble flow was a few millimeters. $P R F=8 \mathrm{kHz}, N=20, I_{\text {spta }}=450 \mathrm{~mW} / \mathrm{cm}^{2}$. 

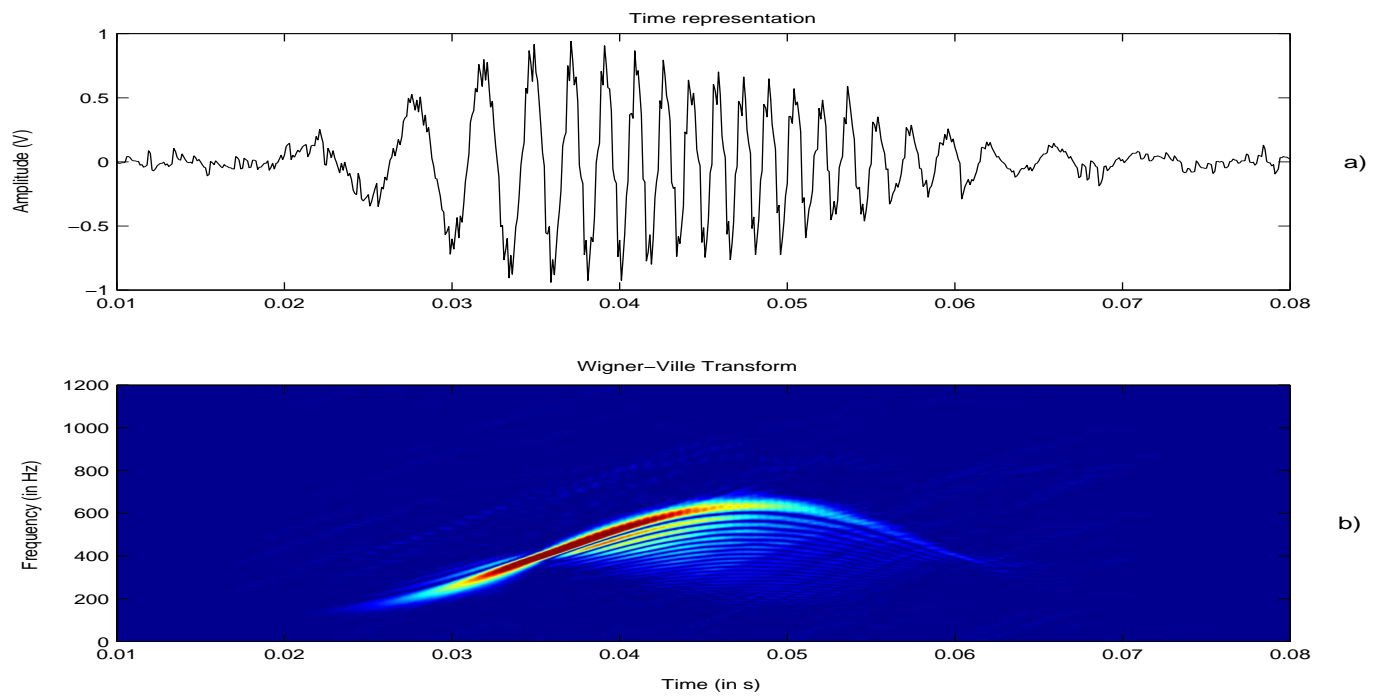

b)
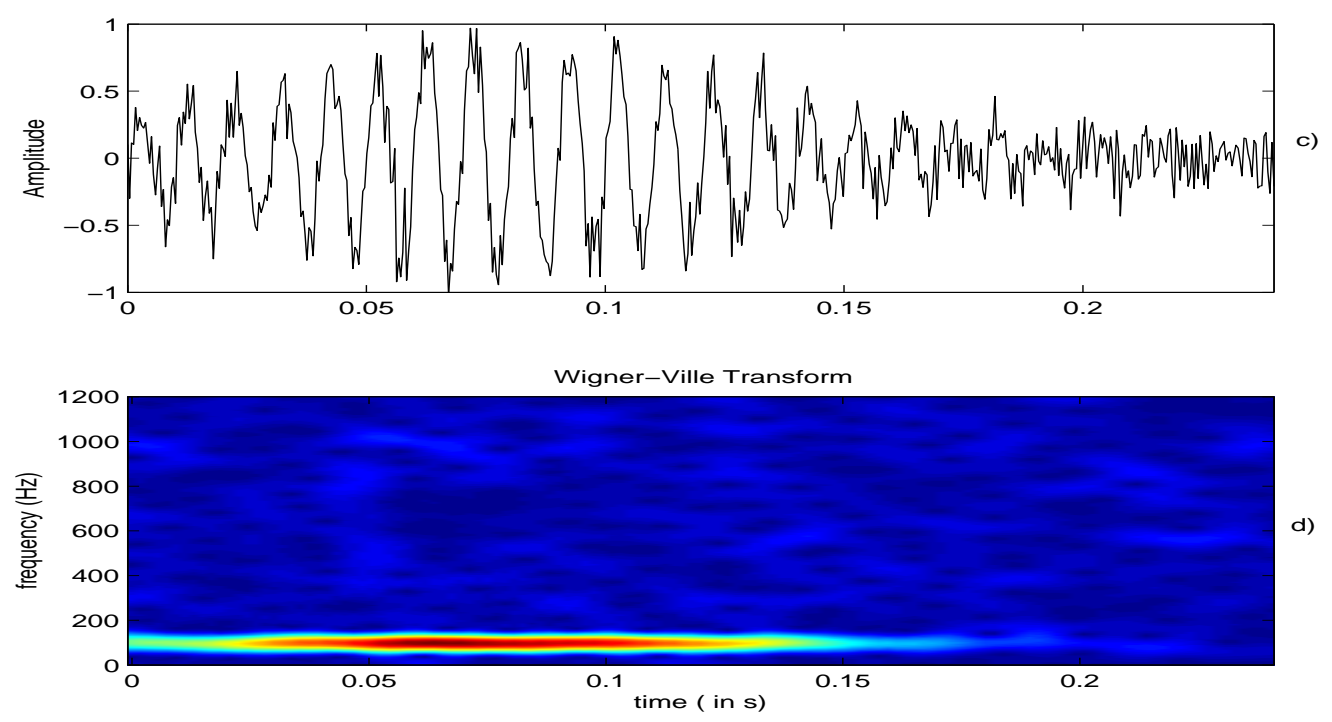

Fig. 8. In vitro experiment in water without a skull. a) time representation of an MES, b) Time frequency representation (Wigner-Ville Transform for improved time-frequency resolution) of a Doppler signal. MES were obtained by means of a TCD system (Atys Medical) $(P R F=8 \mathrm{kHz}$, $N=20$ cycles, $\left.f=2 \mathrm{MHz}, I_{\text {spta }}=450 \mathrm{~mW} / \mathrm{cm}^{2}\right)$. Gaseous embolus $\left(R_{0} \approx 100 \mu \mathrm{m}\right)$ circulating in a tube filled with water (in vitro experiment depicted in Fig.3) (upper graph), $\Delta_{f} / \Delta_{t}=(600-200) / 0.02=20 \mathrm{kHz} / \mathrm{s}$. c) Time representation of an MES and d) Time frequency representation of an MES. A fat particle $\left(R_{0} \approx 300 \mu \mathrm{m}\right)$ (piece of pork) circulating in a tube filled with water (in vitro experiment depicted in Fig.3) (bottom graph), $\Delta_{f} / \Delta_{t} \approx 0$ $\mathrm{Hz} / \mathrm{s}$. The speed of the fluid was identical for all experiments. 


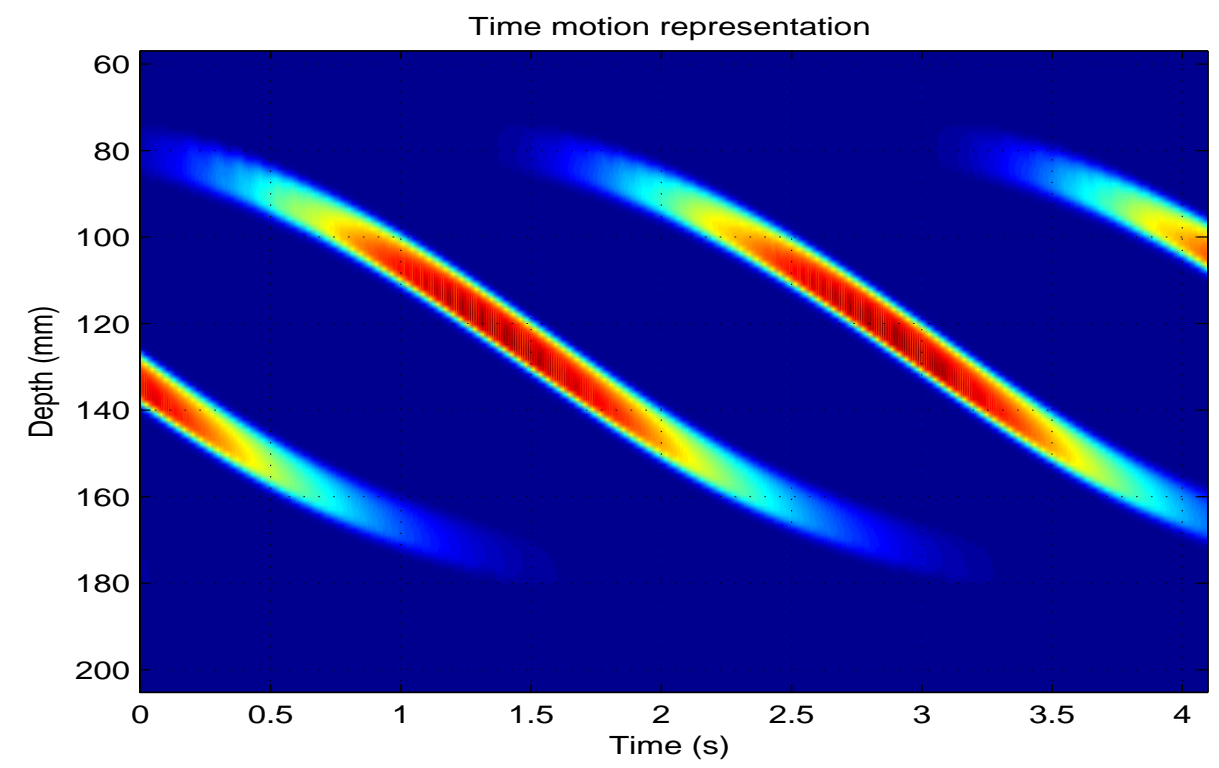

Fig. 9. Time-depth representation of four gaseous emboli (evaluated radius of $\approx 200 \mu \mathrm{m}$ ) circulating in water obtained with the MCMG system as described in the experimental setup in Fig. 4). $f=2 \mathrm{MHz}, P R F=1 \mathrm{kHz}, N=25, P a=500 \mathrm{kPa} . \Delta_{D} / \Delta_{t}=(180-80) /(3) \approx 30$ $\mathrm{mm} / \mathrm{s}$. Note that the thickness of the vertical signature corresponds to $c t / 2=c N / f / 2=$ $1540 \mathrm{~m} / \mathrm{sx} 25 / 2 \mathrm{MHz} / 2 \approx 10 \mathrm{~mm}$. 


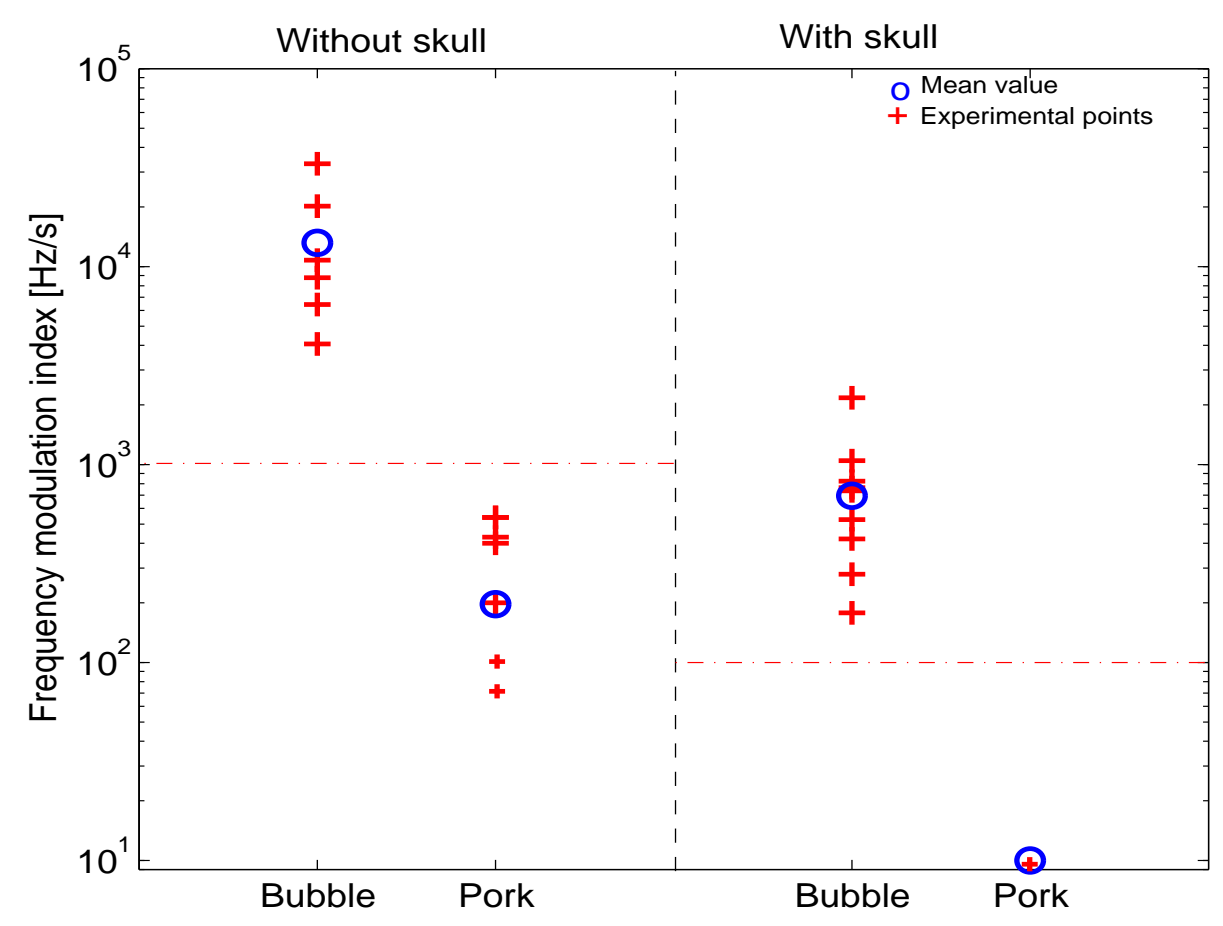

Fig. 10. Frequency modulation index (FMI) for gaseous and solid emboli in the presence or absence of a skull in water $(\eta=1 \mathrm{mPa})$. These measurements were obtained from the experimental setup illustrated in Fig. 4. $+=$ one measurement (several crosses can be superimposed), $o=$ mean of all measurements. $I_{\text {spta }}=450 \mathrm{~mW} / \mathrm{cm}^{2}, N=20, P R F=8 \mathrm{kHz}$. 